\title{
Dual-energy CT with virtual monoenergetic images to improve the visualization of pancreatic supplying arteries: the normal anatomy and variations
}

Hong-wei Liang ${ }^{1}$, Yang Zhou ${ }^{1}$, Zhi-wei Zhang ${ }^{1}$, Gao-wu Yan² ${ }^{2}$ Si-lin Du' ${ }^{1}$ Xiao-hui Zhang ${ }^{1}$, Xin-you Li ${ }^{1}$, Fa-jin Lv ${ }^{1}$, Qiao Zheng ${ }^{1 *}$ and Yong-mei Li ${ }^{1 *}$ (1)

\begin{abstract}
Background: Pancreatic ductal adenocarcinoma (PDAC) remains a malignancy with poor prognosis, appropriate surgical resection and neoadjuvant therapy depend on the accurate identification of pancreatic supplying arteries. We aim to evaluate the ability of monoenergetic images (MEI [+]) of dual-energy CT (DECT) to improve the visualization of pancreatic supplying arteries compared to conventional polyenergetic images (PEI) and investigate the implications of vascular variation in pancreatic surgery and transarterial interventions.

Results: One hundred patients without pancreatic diseases underwent DECT examinations were retrospectively enrolled in this study. The signal-to-noise ratio (SNR) and contrast-to-noise ratio (CNR) at 40-keV MEl (+) were significantly higher than those of PEI $(p<0.05)$. All subjective MEI $(+)$ scores were significantly higher than those of PEI $(p<0.05)$. The visualization rates were significantly higher for posterior superior pancreaticoduodenal artery (PSPDA), anterior and posterior inferior pancreaticoduodenal artery (AIPDA, PIPDA), anterior and posterior pancreaticoduodenal arcade (APAC, PPAC), transverse and caudal pancreatic artery (TPA, PCA) at 40-keV MEI (+) than those of PEI $(p<0.05)$. However, there were no significant differences for visualizing anterior superior pancreaticoduodenal artery (ASPDA), inferior pancreaticoduodenal artery (IPDA), dorsal and magnificent pancreatic artery (DPA, MPA) between 40-keV MEI ( + ) and PEI ( $p>0.05$ ). Four types of variations were observed in the origin of DPA and three to five types in the origin of PSPDA, AIPDA and PIPDA.

Conclusions: $40-\mathrm{keV}$ MEI (+) of DECT improves the visualization and objective and subjective image quality of pancreatic supplying arteries compared to PEl. Pancreatic supplying arteries have great variations, which has important implications for preoperative planning of technically challenging surgeries and transarterial interventions.
\end{abstract}

Keywords: Pancreatic supplying arteries, Dual-energy CT, Virtual monoenergetic images

*Correspondence: 18883936573@163.com; lymzhang70@aliyun.com 1 Department of Radiology, The First Affiliated Hospital of Chongqing Medical University, No. 1 Youyi Road, Yuzhong District, Chongqing 400016, China

Full list of author information is available at the end of the article

\section{Key points}

- 40-keV MEI (+) of DECT improves visualization of pancreatic supplying arteries compared to PEI.

- 40-keV MEI (+) of DECT improves objective and subjective image quality of pancreatic supplying arteries compared to PEI. 
- The normal anatomy and variations in pancreatic supplying arteries have important implications for technically challenging surgeries and transarterial interventions.

\section{Introduction}

Pancreatic ductal adenocarcinoma (PDAC) is the fourth leading cause of cancer deaths in the USA, with an overall survival rate of $6-7 \%[1,2]$. Complete surgical resection (R0) is the only curative option to obtain long-term survival $[3,4]$. For unresectable pancreatic cancer, transarterial chemotherapies have been applied as a neoadjuvant therapy [5-7]. Appropriate surgical resections and local arterial interventions depend on a comprehensive understanding of pancreatic supplying arteries and their variations.

Multidetector computed tomography (MDCT) with thin-section imaging has been used to assess the peripancreatic arterial anatomy $[8,11]$. However, the polychromatic X-ray beam used in a conventional MDCT is more susceptible to beam hardening artifacts [9]. Due to the relatively low resolution of conventional MDCT, this technique permits the major visceral arteries such as the celiac artery (CA), common hepatic artery (CHA) and superior mesenteric artery (SMA) to be identified but does not provide sufficient information on the anatomy of small pancreatic supplying arteries [8]. The recently introduced third generation dual-energy CT (DECT) is the latest tube-based DECT solution, which allows for the reconstruction of monoenergetic images (MEI $[+]$ ) by using the noise-optimized monoenergetic algorithm [11]. MEI $(+)$ approximates the image obtained by monoenergetic X-rays, in which iodine attenuation increases as the energy approximates the iodine k-edge $(33.2 \mathrm{keV})$, so as to acquire higher vessel contrast $[3,13]$. Nagayama and Beer have demonstrated the better visualization at 40-keV MEI $(+)$ in the peripancreatic arteries, but they had only evaluated the major visceral arteries $[3,14]$.

The pancreas receives multiple arterial supplies, leading to a wide variation in its arterial blood distribution, may alter the surgical plan and cause complications in transarterial interventions [7]. It is, therefore, crucial to obtain accurate information on both normal anatomy and variations in pancreatic supplying arteries, but sometimes MDCT cannot provide sufficient information on the pancreatic blood distribution. In such cases, DECT can provide a detailed and accurate assessment. However, the performance of DECT in demonstration of pancreatic supplying arteries and their variations have been scantly evaluated.

The aim of this study is to evaluate the ability of 40-keV MEI (+) of DECT to improve the visualization of pancreatic supplying arteries compared to PEI and to discuss the implications of the normal anatomy and variations in pancreatic supplying arteries for pancreatic surgery and interventional therapy.

\section{Materials and methods}

This retrospective study received institutional review board approval; the requirement for written informed consent was waived.

\section{Patients}

We identified 112 consecutive patients without pancreatic diseases who accepted contrast-enhanced abdominal DECT examinations on a dual-source DECT scanner (SOMATOM Force, Siemens Healthineers) between July 2020 and April 2021. Overall, 12 of 112 patients were excluded for one of the following reasons: vascular obstruction or severe stenosis in the CA, SMA, CHA $(\mathrm{n}=7)$, comorbid renal dysfunction resulted in a reduction in contrast medium protocol $(\mathrm{n}=3)$, and unstable breath-holding $(n=2)$, for a final cohort size of 100 patients (Fig. 1).

\section{CT examinations}

Image data were acquired on a DECT scanner in dualenergy mode through two X-ray tubes with different $\mathrm{kV}$ tube voltages (tube A, $100 \mathrm{kV}$; tube B, Sn $150 \mathrm{kV}$ ), using a tin filter for the high-voltage tube. Settings for both scanners were as follows: collimation $128 \times 0.6 \mathrm{~mm}$; rotation time $0.5 \mathrm{~s}$; pitch 0.6 ; reference tube current time product for the A tube, $260 \mathrm{mAs}$. Automatic exposure control (CAREDose4D) was used in all scans. Images were obtained in a craniocaudal direction from the hepatic dome to bilateral anterior superior iliac spine.

A non-ionic contrast agent (Ultravist 370, Bayer Healthcare; or Iopamiro 370, Bracco Healthcare) was injected at a dose of $1.2 \mathrm{~mL} / \mathrm{kg}$ and at a flow rate of $3.5-5 \mathrm{~mL} / \mathrm{s}$ through a peripheral vein of the forearm, followed by a $40-\mathrm{mL}$ saline flush at the same injection rate. Arterial phase scanning was started with a delay of $10 \mathrm{~s}$ after a trigger threshold in the abdominal aorta $(100 \mathrm{HU})$ was reached, portal venous phase scanning was carried out $30 \mathrm{~s}$ after the end of the arterial phase.

\section{Image reconstruction}

Reconstructed CT image data were post-processed on a syngovia workstation (syngo.via, version VB20A; Siemens Healthineers). Standard linear-blended images were reconstructed by applying a blending factor of 0.6 (M_0.6; 60\% of the $100 \mathrm{kV}$ and $40 \%$ of the Sn $150 \mathrm{KV}$ spectrum) to represent a conventional $120 \mathrm{kV}$ impression. MEI (+) was reconstructed at $40 \mathrm{keV}$ using the novel noise-optimized monoenergetic reconstruction 


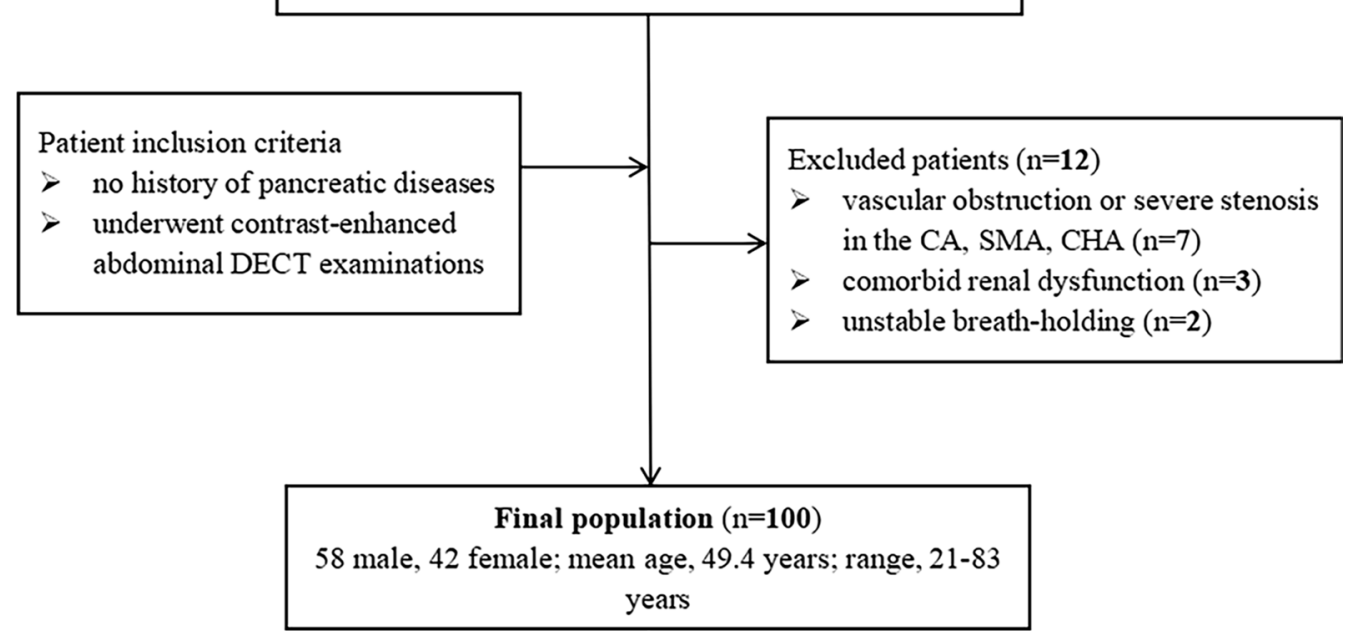

Fig. 1 Flowchart of the included and excluded patients

algorithm. Axial, sagittal, and coronal images were reformatted with a thickness of $1 \mathrm{~mm}$, an increment of $1 \mathrm{~mm}$. Maximum intensity projection (MIP) and volume rendering $(\mathrm{VR})$ were created for both MEI $(+)$ and PEI by a radiologist.

\section{Quantitative analysis}

All these series were analyzed on a syngovia workstation. Objective quantification was performed by a radiologist with 6 years of experience in abdominal imaging. Attenuation of different areas was measured in Hounsfield units (HU), the CT attenuations of pancreatic parenchyma ( $\mathrm{HU}_{\text {pancreas}}$, averaged from normal pancreas parenchyma), peripancreatic arteries $\left(\mathrm{HU}_{\text {artery }}\right.$, averaged from gastroduodenal artery [GDA], ASPDA, and DPA), and retroperitoneal fat $\left(\mathrm{HU}_{\mathrm{fat}}\right)$ were measured by placing circular regions of interest (ROIs). Image noise was quantified as the standard deviation (SD) of $\mathrm{HU}_{\text {fat }}$. For pancreatic attenuation, care was taken to exclude the visible pancreatic duct, vessels, and artifacts. For vessel analysis, firstly, observation and location of pancreatic arteries were performed on the coronal MIP images. If considered visible, the axial images were magnified three times and then used for quantitative analysis, the ROI was placed in the middle of the vessel cavity as far as possible. The above measurements were performed twice and then averaged to confirm data consistency. The quantitative image quality of each object (pancreatic parenchyma, peripancreatic arteries) was calculated with Eqs. $(3,14)$ :

$$
\begin{aligned}
& \mathrm{CNR}_{\text {object }}=\left(\mathrm{HU}_{\text {object }}-\mathrm{HU}_{\text {pancreas }}\right) / \mathrm{SD}_{\text {fat }} \\
& \mathrm{SNR}_{\text {object }}=\mathrm{HU}_{\text {object }} / \mathrm{SD}_{\mathrm{fat}}
\end{aligned}
$$

\section{Qualitative analysis}

Two radiologists with 6 and 10 years of experience of abdominal imaging, respectively, independently reviewed the axial and reformatted images. They were blinded to any patient clinical information and reconstruction parameters. PEI and MEI (+) obtained from the artery phases were separately assessed in random order. Based on the findings of previous reports [10, $11,15]$, the ASPDA, PSPDA, IPDA AIPDA, PIPDA, DPA, MPA, TPA, CPA, APAC and PPAC were identified, and the frequency of visualization and variations in these arteries were recorded. Five-point scales were used to assess visualization of arteries: 5 (excellent, the origin of the vessel is identified and its anatomic course is clearly traced; vessel strengthened obviously), 4 (good, the origin of the vessel is identified, and its anatomic course is traced with reasonable certainty; vessel strengthened well), 3 (average, the origin of the vessel is identified, but only the proximal part of its anatomic course is traced; the edge vessel is not sharp or fuzzy), 2 (suboptimal, the origin of the vessel is identified; the vessels are slender), 1 (poor, the origin of the vessel is not identified; the vessels are not clear). The frequency of visualization and variation in the arteries were assessed by two radiologists, and the final decisions were reached by consensus. 


\section{Statistical analysis}

Statistical analysis was performed using SPSS version 26.0 (IBM Cor, 2013) and GraphPad Prism V5. The Kolmogorov-Smirnov test was applied to assess the normality of data distribution. The continuous variables were expressed as means $\pm \mathrm{SD}$. Chi-square test was applied to assess differences in visualization rate of pancreatic supplying arteries between MEI (+) and PEI. An independent $t$ test was performed to compare the quantitative parameters. A Mann-Whitney U test was performed to compare the qualitative parameters. The inter-reader agreement for qualitative analysis was assessed using Cohen's kappa ( $>0.81$, excellent; 0.61-0.80, substantial; $0.41-0.60$, moderate; $0.21-0.40$, fair; and $<0.20$, poor agreement). Differences of $p<0.05$ were considered statistically significant.

\section{Results}

\section{Objective image parameters}

40-keV MEI (+) significantly improved the CNR and SNR of the GDA, ASPDA, and DPA compared with the PEI $(p<0.05)$. For all vessels analyses, compared with PEI, the CNR and SNR of the GDA obtained at 40-keV MEI (+) increased by $60.03 \%$ and $50.76 \%$, respectively $(p<0.001)$. The CNR and SNR of the ASPDA obtained at $40-\mathrm{keV}$ MEI (+) increased by $41.60 \%$ and $38.66 \%$, respectively $(p<0.05)$. The CNR and SNR of the DPA obtained at $40-\mathrm{keV}$ MEI $(+)$ increased by $42.43 \%$ and $39.20 \%$, respectively $(p<0.001)$ (Fig. 2).

For pancreatic parenchyma, the SNR obtained at 40-keV MEI $(+)$ increased by $58.14 \%$ compared with PEI $(p<0.001)$. The image noise at $40-\mathrm{keV}$ MEI $(+)$ was significantly higher than that of PEI $(p<0.001)$. Full results of the quantitative analysis are shown in Table 1.
Table 1 Quantitative analysis

\begin{tabular}{lllr}
\hline & 40-keV MEI(+) & PEI & $p$ \\
\hline Image noise & $22.49 \pm 3.24$ & $14.32 \pm 1.49$ & $<0.001$ \\
CNR & & & \\
GDA & $23.22 \pm 5.95$ & $12.43 \pm 3.09$ & $<0.001$ \\
ASPDA & $14.67 \pm 4.31$ & $8.88 \pm 2.83$ & 0.011 \\
DPA & $15.81 \pm 4.66$ & $9.49 \pm 2.63$ & $<0.001$ \\
SNR & & & \\
GDA & $34.78 \pm 7.04$ & $19.74 \pm 3.46$ & $<0.001$ \\
ASPDA & $26.22 \pm 5.54$ & $16.18 \pm 3.08$ & $<0.001$ \\
DPA & $27.31 \pm 5.82$ & $16.74 \pm 3.07$ & $<0.001$ \\
Pancreatic parenchyma & $11.56 \pm 2.78$ & $7.31 \pm 1.31$ & $<0.001$ \\
\hline
\end{tabular}

Data shown are mean \pm standard deviation

CNR, contrast-to-noise ratio; SNR, signal-to-noise ratio; GDA, gastroduodenal artery; ASPDA, anterior superior pancreaticoduodenal artery; DPA, dorsal pancreatic artery

\section{Subjective image quality}

Compared with PEI, 40-keV MEI (+) provided significantly higher subjective scores for all the pancreatic supplying arteries $(p<0.05)$ (Table 2). There was moderate to substantial inter-reader agreement for $40-\mathrm{keV}$ MEI $(+) \quad($ kappa $=0.56-0.79)$ and PEI (kappa $=0.55-0.77)$, respectively.

\section{The frequency of visualization of vessels}

The display rates were significantly higher for PSPDA, AIPDA, PIPDA, APAC, PPAC, TPA, and PCA at 40-keV MEI $(+)$ than in PEI ( $96 \%$ vs $88 \%$; $96 \%$ vs $87 \%$; $93 \%$ vs $86 \%$; $60 \%$ vs $45 \%$; $53 \%$ vs $38 \%$; $84 \%$ vs $72 \%$; $64 \%$ vs $49 \%$; $p<0.05)$. However, there were no significant differences for visualizing ASPDA, IPDA, DPA, and MPA between $40-\mathrm{keV}$ MEI $(+)$ and PEI ( $100 \%$ vs $99 \%$; $51 \%$ vs $48 \%$; $92 \%$ vs $86 \% ; 87 \%$ vs $82 \% ; p>0.05$ ) (Table 3 ).
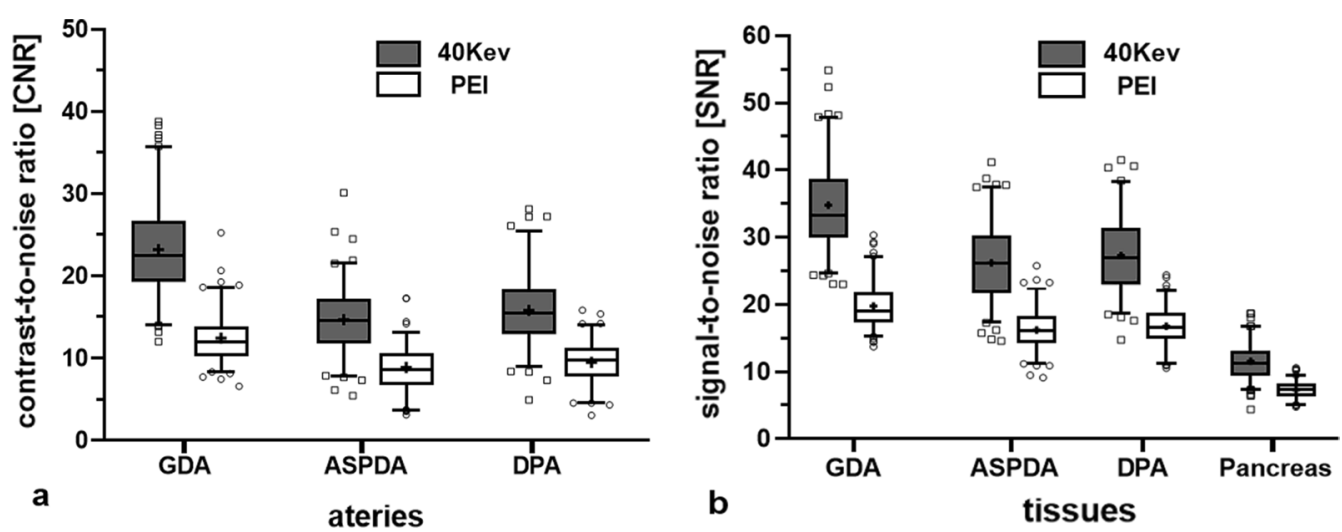

Fig. 2 Box-and-whisker plots of the differences in CNR (a) and SNR (b) between 40-keV MEI (+) and PEl. The box indicates the 25- and 75-quartile; the horizontal line indicates the median and the cross indicates the mean. Whiskers show the 5 and 95 percentiles; outliers are indicated by squares and circles 
Table 2 Qualitative analysis

\begin{tabular}{|c|c|c|c|c|c|c|c|c|c|}
\hline & ASPDA & PSPDA & IPDA & AIPDA & PIPDA & DPA & MPA & TPA & PCA \\
\hline 40-keV MEI (+) & $4.19 \pm 0.82^{*}$ & $3.58 \pm 0.97^{*}$ & $4.18 \pm 0.69^{*}$ & $3.42 \pm 0.79^{*}$ & $2.97 \pm 0.77^{*}$ & $4.10 \pm 0.79^{*}$ & $3.57 \pm 0.73^{*}$ & $3.44 \pm 0.77^{*}$ & $2.88 \pm 0.54^{*}$ \\
\hline PEI & $3.34 \pm 0.85$ & $2.99 \pm 0.80$ & $3.71 \pm 0.79$ & $2.86 \pm 0.69$ & $2.50 \pm 0.69$ & $3.45 \pm 0.71$ & $2.89 \pm 0.61$ & $2.69 \pm 0.74$ & $2.35 \pm 0.49$ \\
\hline Kappa & 0.74 & 0.64 & 0.65 & 0.73 & 0.62 & 0.72 & 0.67 & 0.75 & 0.71 \\
\hline
\end{tabular}

Data shown are mean \pm standard deviation

*Scores for 40-keV MEI $(+)>$ scores for PEI $(p<0.05)$

ASPDA, anterior superior pancreaticoduodenal artery; PSPDA, posterior superior pancreaticoduodenal artery; IPDA, inferior pancreaticoduodenal artery; AIPDA, anterior inferior pancreaticoduodenal artery; PIPDA, posterior inferior pancreaticoduodenal artery; DPA, dorsal pancreatic artery; MPA, magnificent pancreatic artery; TPA, transverse pancreatic artery; PCA, caudal pancreatic artery

Table 3 The frequency of visualization of arteries

\begin{tabular}{llll}
\hline Artery & 40-keV MEI (+) (\%) & PEI (\%) & $\boldsymbol{P}$ \\
\hline ASPDA & 100 & 99 & 0.316 \\
PSPDA & 96 & 88 & $0.037^{*}$ \\
IPDA & 51 & 48 & 0.671 \\
AIPDA & 96 & 87 & $0.022^{*}$ \\
PIPDA & 93 & 86 & $0.046^{*}$ \\
APAC & 60 & 45 & $0.034^{*}$ \\
PPAC & 53 & 38 & $0.033^{*}$ \\
DPA & 92 & 86 & 0.175 \\
MPA & 87 & 82 & 0.329 \\
TPA & 84 & 72 & $0.041^{*}$ \\
PCA & 64 & 49 & $0.032^{*}$
\end{tabular}

*40-keV MEI (+) $>$ PEl in the frequency of visualization of arteries $(p<0.05)$ ASPDA, anterior superior pancreaticoduodenal artery; PSPDA, posterior superior pancreaticoduodenal artery; IPDA, inferior pancreaticoduodenal artery; AIPDA, anterior inferior pancreaticoduodenal artery; PIPDA, posterior inferior pancreaticoduodenal artery; APAC, anterior pancreaticoduodenal arcade; PPAC, posterior pancreaticoduodenal arcade; DPA, dorsal pancreatic artery; MPA, magnificent pancreatic artery; TPA, transverse pancreatic artery; PCA, caudal pancreatic artery

\section{The normal anatomy and variations in vessels at $40-\mathrm{keV}$ MEl (+) \\ Superior pancreaticoduodenal artery (ASPDA and PSPDA)}

The ASPDA originated as minor terminal branch of the GDA in all the cases, in 63 cases, anastomoses between the ASPDA and AIPDA were apparent, forming a standard single anterior arcade $(n=60)$ (Fig. $3 b)$, two arcades $(n=10)$, and an anastomotic branch with the DPA (prepancreatic Kirk arcade) $(\mathrm{n}=13)$.

Three types of variations were observed in the origin of PSPDA. The PSPDA arose from (a) GDA ( $n=92$; Fig. 3c); (b) aberrant right hepatic artery (a/r RHA) from SMA $(\mathrm{n}=2)$; (c) PHA $(\mathrm{n}=2)$. The collateral branches of PSPDA were visualized: retroduodenal arteries $(n=28)$; in 59 cases, the anastomoses with the PIPDA were appreciable, with a standard single posterior arcade $(n=53)$ (Fig. 4b), two arcades $(n=6)$ and an anastomotic branch with the DPA (retropancreatic arcade) $(\mathrm{n}=8)$; and in 4 cases, it had both the prepancreatic and retropancreatic arcade.

Inferior pancreaticoduodenal artery (IPDA, AIPDA, PIPDA) The IPDA predominantly originated from SMA and bifurcated into the AIPDA and PIPDA $(n=35)$. Other origins of the IPDA were from a common trunk with the first jejunal artery (JA) forming a pancreatico-duodenojejunal (PDJ) trunk $(\mathrm{n}=15$; Fig. $5 \mathrm{~d})$ and from the middle colon artery $(\mathrm{n}=1)$.

In the cases which did not have an IPDA, four to five types of variations were observed in the origin of AIPDA and PIPDA (Table 4). The AIPDA arose from (a) SMA $(\mathrm{n}=27) ;(\mathrm{b})$ DPA $(\mathrm{n}=4)$; (c) first JA $(\mathrm{n}=12) ;(\mathrm{d}) \mathrm{a} / \mathrm{r}$ RHA $(\mathrm{n}=1) ;(\mathrm{e})$ CHA $(\mathrm{n}=1)$. And the PIPDA arose from (a) SMA $(n=24)$; (b) DPA $(n=7)$; (c) first or second JA $(\mathrm{n}=5)$; (d) a/r RHA $(\mathrm{n}=2)$.

\section{Dorsal pancreatic artery (DPA)}

The DPA supplied the body of the pancreas, which had the greatest variability. The DPA originated from (a) splenic artery (SPA) $(n=62$; Fig. 5a); (b) SMA ( $n=14$; Fig. 5c); (c) CHA ( $n=11$; Fig. 5b); (d) CA $(n=5)$. At the lower edge of the pancreas, DPA bifurcated into the right branch and left terminal branches, which formed an inverted " $\mathrm{T}$ " pattern branching $(\mathrm{n}=25)$. The left branch corresponded to the TPA, while the right branch constituted the prepancreatic Kirk arcade with the ASPDA $(n=13)$ and the retropancreatic arcade with the PSPDA $(\mathrm{n}=8)$.

\section{Transverse pancreatic artery (TPA)}

The TPA arose from (a) DPA and represented its left branch ( $\mathrm{n}=71$; Fig. 5a); (b) the left branch of ASPDA $(\mathrm{n}=8) ;(\mathrm{c})$ PDJ trunk $(\mathrm{n}=3)$. There were anastomoses between the TPA and MPA $(n=35)$ and the first JA $(n=2)$. 


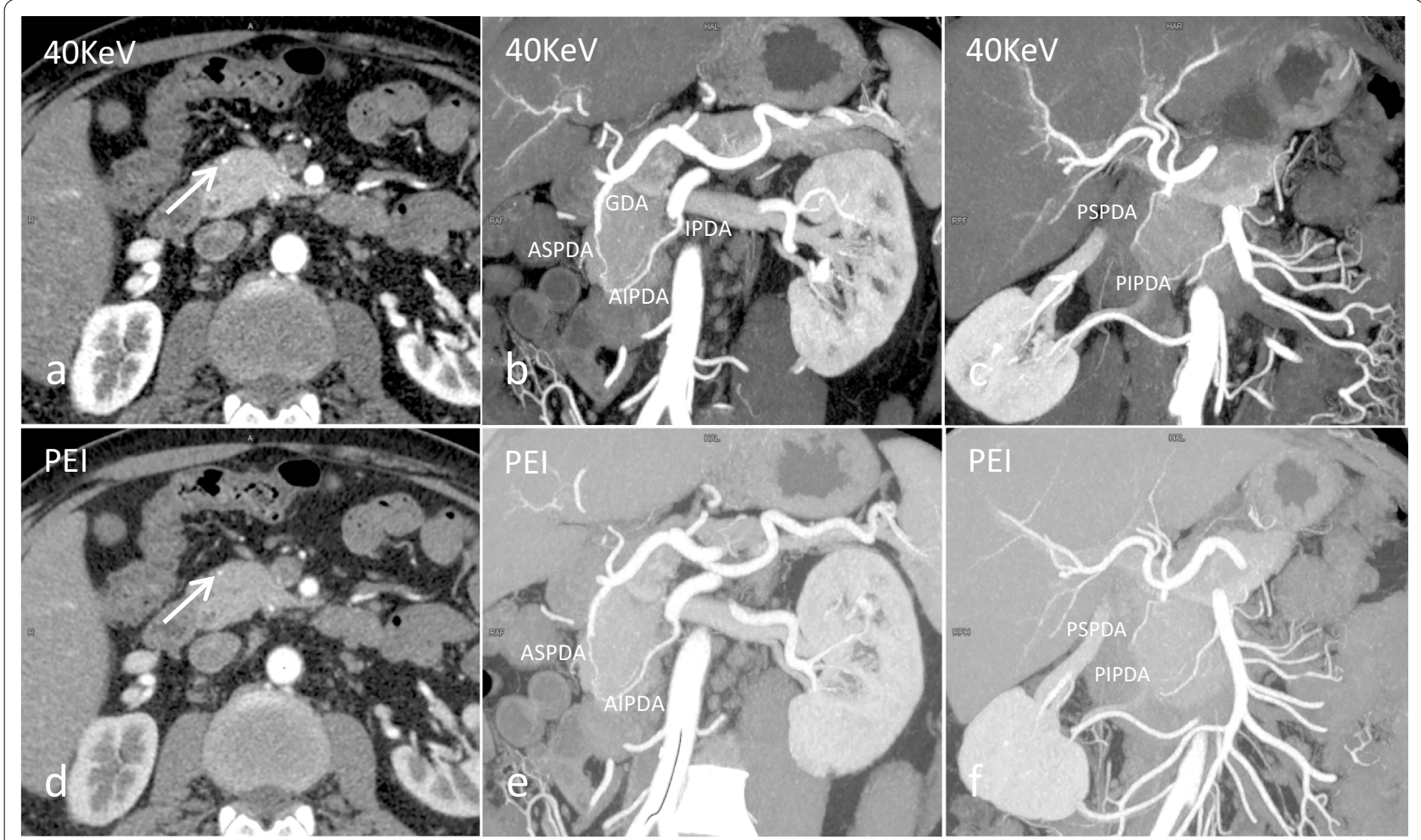

Fig. 3 The upper $(\mathbf{a}-\mathbf{c})$ and lower $(\mathbf{d}-\mathbf{f})$ rows show the 40-keV MEI $(+)$ and PEI images, respectively. a The axial image shows that the anterior superior pancreaticoduodenal artery (ASPDA) runs along the anterior and lateral surface of the pancreatic head (arrow). $\mathbf{b}$ Coronal reformatted image shows the ASPDA originating from gastroduodenal artery (GDA), and anastomosing inferiorly with anterior inferior pancreaticoduodenal artery (AIPDA) to form the standard anterior pancreaticoduodenal arcade. c Coronal reformatted image shows the posterior superior pancreaticoduodenal artery (PSPDA) and the posterior inferior pancreaticoduodenal artery (PIPDA). The visualization is better on the 40-keV MEI (+), compared to the PEI

\section{Discussion}

This study demonstrated that 40-keV MEI (+) of DECT provided significantly better objective and subjective image quality in depicting pancreatic supplying arteries relative to conventional PEI. The visualization rates were significantly higher for PSPDA, AIPDA, PIPDA, APAC, PPAC, TPA, and PCA at 40-keV MEI (+) than in PEI. Furthermore, pancreatic supplying arteries have great variabilities, which have important implications for preoperative planning of technically challenging surgeries and transarterial interventions.

In previous studies, some authors have indicated that MEI (+) reconstructions can yield improvement in the visualization of the major visceral arteries [3, 12, 14]. Nagayama and Beer have demonstrated the higher SNR and $\mathrm{CNR}$ at $40-\mathrm{keV}$ MEI $(+)$ in peripancreatic arteries (CA, CHA, SMA) [3, 14]. These studies favored low $\mathrm{keV}$ MEI (+) in terms of subjective and objective image parameters. However, the performance of DECT in demonstration of small pancreatic supplying arteries and their variations had been scantly investigated. In this study, we evaluated the ability of $40-\mathrm{keV}$ MEI (+) of DECT to improve visualization of pancreatic supplying arteries compared with PEI and analyzed their normal anatomy and variations, using a much larger patient cohort.

The visualization of the enhanced vessels is mainly dependent on the image noise and the degree of vascular enhancement. However, reducing noise requires to increase radiation dose; improving the degree of vascular enhancement requires to increase the contrast dose and flow rate. However, previous study suggested that the fast injection rate increases the risk of extravasation and hyperosmolarity of contrast material [16]. CNR is the main objective parameter of vessel contrast. DECT MEI ( + ) can improve the degree of vascular enhancement without increasing the dose of radiation and contrast agent, so as to improve the CNR of enhanced vessels [17]. In our study, the CNR of GDA, ASPDA, and DPA at $40-\mathrm{keV}$ MEI $(+)$ increased by $60.03 \%, 41.60 \%$, and $42.43 \%$, respectively, compared with PEI.

Image noise is another factor affecting the visualization of the pancreatic supplying arteries. The objective image noise of $40-\mathrm{keV}$ MEI $(+)$ is higher than that 


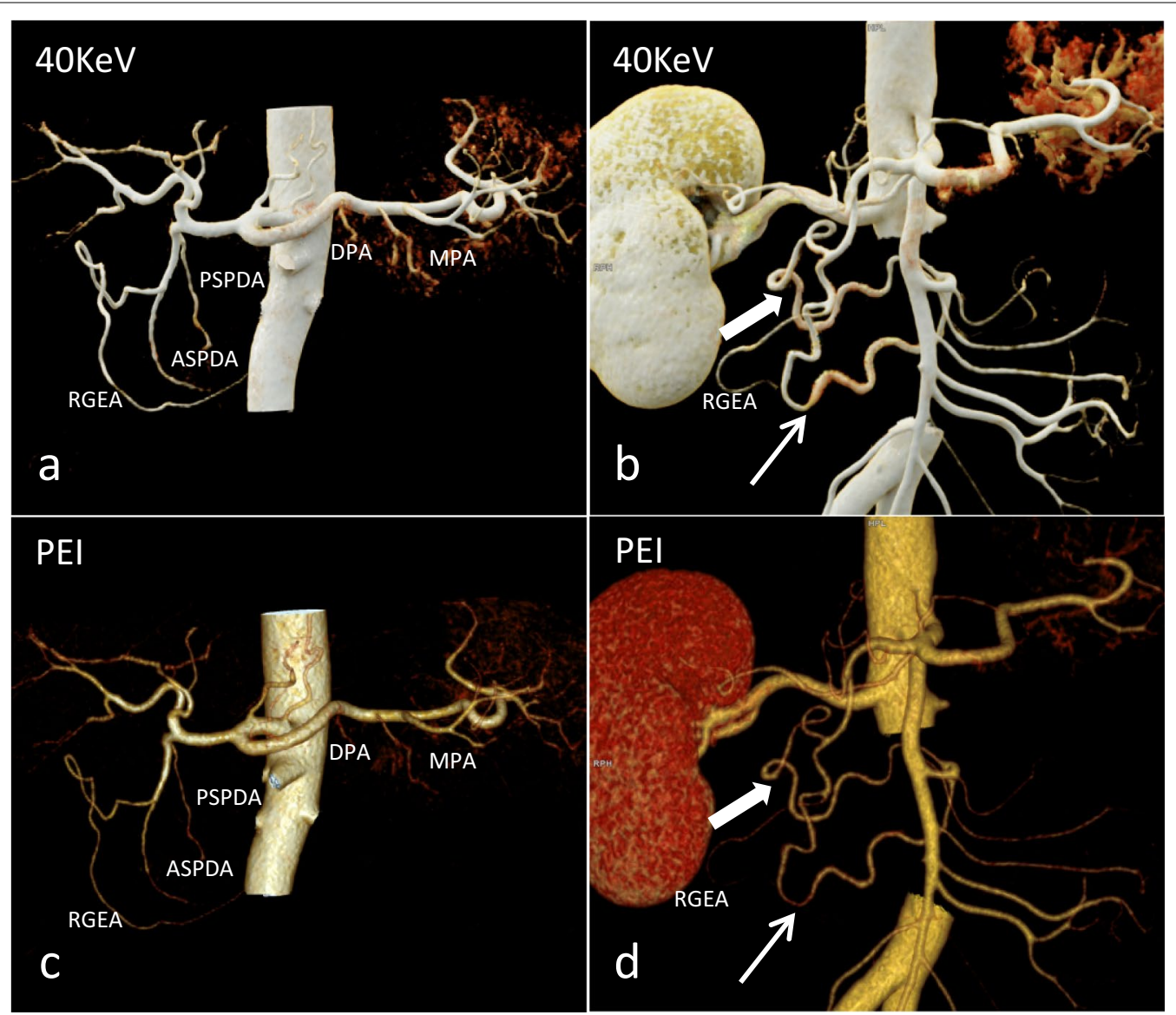

Fig. 4 The upper (a, b) and lower (c, d) rows show the 40-keV MEI (+) and PEl images, respectively. a Volume Rendering Technology (VRT) of contrast-enhanced CT angiography shows the anterior superior pancreaticoduodenal artery (ASPDA), posterior superior pancreaticoduodenal artery (PSPDA), dorsal pancreatic artery (DPA) and magnificent pancreatic artery (MPA). b VRT shows standard anterior (thin arrow) and posterior (thick arrow) pancreaticoduodenal arcade. The visualization is better on the 40-keV MEI (+), compared to the PEl. RGEA= Right gastroepiploic artery

of PEI in our study, which in accordance with recent investigations that have shown the increased noise for lower energy levels [12, 14]. However, compared with standard monoenergetic algorithm, a novel noise-optimized monoenergetic algorithm had been introduced to overcome this limitation in the third generation DECT. This technique performs a spatial frequencybased recombination that reduces the image noise of lower energies and improves image contrast at higher energies to obtain the best image quality [3, 14]. In addition, although image noise is often used as an image quality indicator, the consideration for the SNR level is also necessary. Increased SNR could necessarily guarantee improved image quality if the absolute noise level is clinically acceptable [12]. In our study, the SNR of pancreatic parenchyma, GDA, ASPDA, and DPA in 40-keV MEI (+) increased by $58.14 \%, 50.76 \%$, 38.66\%, and $39.20 \%$, respectively, compared with PEI. Moreover, an advanced modeled iterative reconstruction was applied for DECT to suppress the image noise and improve image quality $[18,19]$. All these reasons may contribute to the better image quality at 40-keV MEI $(+)$ than PEI.

Improving the visualization of pancreatic supplying arteries is important for some planning limited surgery, such as duodenum-preserving resection of the pancreatic head [20]. In order to perform surgical resection of the pancreatic head safely, it is crucial to clearly depict the anatomy of the pancreaticoduodenal arcades [8]. In addition, familiarization with the normal anatomy and variations in pancreatic supplying arteries is important in planning transarterial interventions. Tanaka et al. demonstrated that the optimal drug distribution in arterial infusion chemotherapy for advanced PDAC was related with proper placement of the catheter [21]. The catheter placement in arterial infusion depends largely on the precise knowledge about the blood distribution and variations because the arteries supply to each region of the 

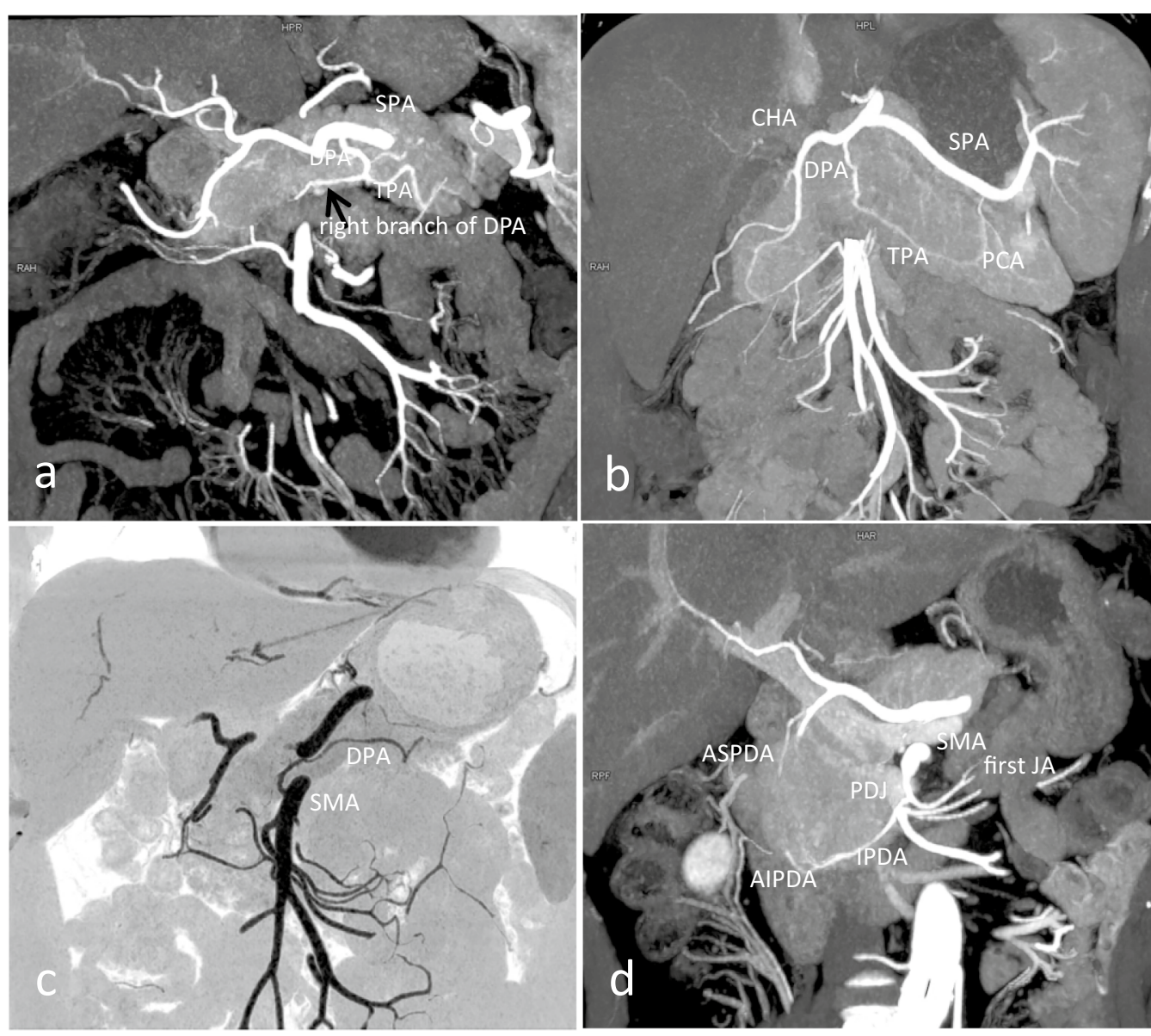

Fig. 5 The normal anatomy and variations in pancreatic supplying arteries. a MIP shows the dorsal pancreatic artery (DPA) originating from splenic artery (SPA), bifurcates into the right branch and the transverse pancreatic artery (TPA), which forms an inverted "T" pattern branching. $\mathbf{b}$ The DPA originates from the common hepatic artery (CHA), bifurcates into the TPA, and then anastomoses with caudal pancreatic artery (PCA). c Minimum intensity projection (MinIP) shows the DPA originating from the superior mesenteric artery (SMA). d MIP shows the inferior pancreaticoduodenal artery (IPDA) originating from the first jejunal artery (JA) to form the pancreatico-duodeno-jejunal (PDJ) trunk

Table 4 The major origin of IPDA, AIPDA, and PIPDA

\begin{tabular}{lll}
\hline Origin of IPDA & Variations in AIPDA and PIPDA origin & $\%(\mathbf{n})$ \\
\hline SMA & AIPDA and PIPDA from IPDA bifurcation & $35 \%$ \\
& & $(35 / 100)$ \\
From a common trunk (PDJ) with first jejunal artery (JA) & AIPDA and PIPDA from IPDA bifurcation & $14 \%$ \\
middle colon artery & AIPDA and PIPDA from IPDA bifurcation & $(14 / 100)$ \\
Absent IPDA & Separate origin of AIPDA and PIPDA from SMA & $1 \%(1 / 100)$ \\
Absent IPDA & & $22 \%$ \\
Absent IPDA & AIPDA from first JA and PIPDA from SMA & $(22 / 100)$ \\
Absent IPDA & PIPDA from DPA and AIPDA from SMA & $5 \%(5 / 100)$ \\
Absent IPDA & AIPDA from SMA and PIPDA absent & $5 \%(5 / 100)$ \\
\hline
\end{tabular}

IPDA, inferior pancreaticoduodenal artery; AIPDA, anterior inferior pancreaticoduodenal artery; PIPDA, posterior inferior pancreaticoduodenal artery; DPA, dorsal pancreatic artery; SMA, superior mesenteric artery; a/rRCHA, aberrant right hepatic artery; JA, jejunal artery; PDJ, pancreaticoduodenojejunal trunk 
pancreas is different [21]. In our study, except the ASPDA, IPDA, DPA and MPA, the visualization rates of pancreatic supplying arteries at $40-\mathrm{keV}$ MEI (+) were significantly higher than that of PEI. One potential explanation may be that the ASPDA, IPDA, DPA and MPA have a relatively thick luminal diameter and high visualization rate at the conventional PEI, so the visualization rate at $40-\mathrm{keV}$ MEI $(+)$ was not significantly improved $[11,22,23]$.

Pancreas has complex arterial supply, and the anatomy of pancreatic supplying arteries is highly variable, both in origin and distribution [24]. In our study, the display rates were higher for ASPDA and PSPDA at 40-keV MEI $(+)$ than in PEI (100\% vs $99 \%$; $96 \%$ vs $88 \%)$. And high variability was observed in their origin. At 40-keV MEI (+), ASPDA arose from GDA in 100\% and PSPDA in 95.83\% cases, like the previously reported data $(95-100 \%$ and $92.5-96.2 \%$, respectively) $[10,15]$. In the remaining cases, the PSPDA originated from PHA and a/r RHA from SMA in four cases. Generally, blood supply of the superior head of the pancreas is always from the GDA and $\mathrm{CA}$, and Sakuhara et al. suggested that CT during arteriography is not required to confirm the supplying artery in these regions [7]. However, in our study, PSPDA originated from $\mathrm{a} / \mathrm{r}$ RHA from SMA in two cases. It is crucial to be familiar with such arterial patterns before superselective transarterial interventions.

The IPDA was present in $51 \%$ cases at $40-\mathrm{keV}$ MEI (+) in our study, which was higher than the PEI (48\%). At 40-keV MEI (+), IPDA originated from SMA in 35 cases, and PDJ trunk in 15 cases. Other origin of IPDA was the middle colon artery in one case. Knowledge of this rare variation is essential to avoid potential complications during pancreatic surgical and transarterial interventions. The occurrence of AIPDA and PIPDA has been reported by most of the previous studies with an overall incidence of $96-100 \%[10,11]$. In our study, these were found in $96 \%$ and $93 \%$ cases at $40-\mathrm{keV} \mathrm{MEI}(+)$, respectively. In the cases which did not have an IPDA, the other sources of origin of AIPDA in our study were SMA in 27 cases, first JA in 12 cases, DPA, a/r RHA and CHA in 6 cases. On the other hand, the PIPDA arose from SMA in 24 cases, DPA in 7 cases, first or second JA and a/r RHA in 7 cases. The DPA has the greatest variability of all the arteries supplying the pancreas. In our study, the DPA was present in $92 \%$ cases at $40-\mathrm{keV}$ MEI (+), which was higher than the PEI (86\%). Doppman et al. suggested that the DPA predominantly originates from the SPA and to supply the body of the pancreas [25]. In our study, the DPA has been found to arise from SPA in 62 cases, which was mostly in agreement with theirs, other origins were SMA in 14 cases, CHA in 11 cases and CA in 5 cases. This result indicated that the body of the pancreas is supplied by the SMA when the DPA originates from the SMA.
This study has several limitations. Firstly, image analysis was based on conventional PEI and 40-keV MEI $(+)$ reconstructions. Other MEI (+), such as $50-\mathrm{keV}$ or 60-keV, were not assessed since previous studies have shown $40-\mathrm{keV}$ was the optimal MEI (+). Secondly, the pancreatic supplying arteries did not have anatomic reference standard in our study. However, we believe that our artery identification was accurate, and it was confirmed that the findings essentially accord with those of previous anatomic and radiological reports. Finally, we did not analyze the influence of pancreatic diseases on pancreatic supplying arteries. Therefore, further investigations should be performed on the relationship between pancreatic diseases and pancreatic supplying arteries.

\section{Conclusion}

In conclusion, 40-keV MEI (+) of DECT substantially improves the visualization and objective and subjective image quality of pancreatic supplying arteries compared to conventional PEI. Accurate preoperative identification with the normal anatomy and variations in pancreatic supplying arteries has important clinical significance for pancreatic surgery and transarterial interventions.

\section{Abbreviations \\ a/r RHA: Aberrant right hepatic artery; AIPDA: Anterior inferior pancreaticodu- odenal artery; APAC: Anterior pancreaticoduodenal arcade; ASPDA: Anterior superior pancreaticoduodenal artery; CA: Celiac artery; CHA: Common hepatic artery; CNR: Contrast-to-noise ratio; DECT: Dual-energy CT; DPA: Dorsal pancre- atic artery; GDA: Gastroduodenal artery; IPDA: Inferior pancreaticoduodenal artery; JA: Jejunal artery; MEI (+): Monoenergetic images; MPA: Magnificent pancreatic artery; PCA: Caudal pancreatic artery; PDJ: Pancreatico-duodeno- jejunal trunk; PEl: Polyenergetic images; PIPDA: Posterior inferior pancrea- ticoduodenal artery; PPAC: Posterior pancreaticoduodenal arcade; PSPDA: Posterior superior pancreaticoduodenal artery; SMA: Superior mesenteric artery; SNR: Signal-to-noise ratio; TPA: Transverse pancreatic artery.}

\section{Authors' contributions}

HWL contributed to acquisition, interpretation of data, draft the work; YZ contributed to design of the work and revised the work; ZWZ contributed to conception of the work and drafted the work; GWY contributed to interpretation of data and revised the work; SLD and XHZ revised the work; XYL and FJL monitored the process of the study; QZ and YML contributed to conception of the work and revised the work. All authors read and approved the final manuscript.

\section{Funding}

This work was funded by the Foundation of the Medicine Scientific key Research Project of Chongqing Municipal Health and Family Planning Commission (No. 2016ZDXM002) and the First Affiliated Hospital of Chongqing Medical University (PYJJ2020-06).

\section{Availability of data and materials}

The datasets used and/or analyzed during the current study are available from the corresponding author on reasonable request.

\section{Declarations}

Ethics approval and consent to participate

The study was approved by the ethics committee of the First Affiliated Hospital of Chongqing Medical University (No.: 2021-176), and individual consent for this retrospective analysis was waived. 


\section{Consent for publication}

Not applicable.

\section{Competing interests}

The authors declare that they have no competing interests.

\section{Author details}

'Department of Radiology, The First Affiliated Hospital of Chongqing Medical University, No. 1 Youyi Road, Yuzhong District, Chongqing 400016, China.

${ }^{2}$ Department of Radiology, Suining Central Hospital, Suining 629000, China.

Received: 13 October 2021 Accepted: 7 January 2022

Published online: 04 February 2022

\section{References}

1. Siegel RL, Miller KD, Jemal A (2019) Cancer statistics, 2019. CA Cancer J Clin 69:7-34. https://doi.org/10.3322/caac.21551

2. Zaky AM, Wolfgang CL, Weiss MJ, Javed AA, Fishman EK, Zaheer A (2017) Tumor-vessel relationships in pancreatic ductal adenocarcinoma at multidetector CT: different classification systems and their influence on treatment planning. Radiographics 37:93-112. https://doi.org/10.1148/rg. 2017160054

3. Nagayama Y, Tanoue S, Inoue T et al (2020) Dual-layer spectral CT improves image quality of multiphasic pancreas $\mathrm{CT}$ in patients with pancreatic ductal adenocarcinoma. Eur Radiol 30:394-403. https://doi.org/10. 1007/s00330-019-06337-y

4. Konstantinidis IT, Warshaw AL, Allen JN et al (2013) Pancreatic ductal adenocarcinoma: is there a survival difference for $\mathrm{R} 1$ resections versus locally advanced unresectable tumors? What is a "true" R0 resection? Ann Surg 257:731-736. https://doi.org/10.1097/SLA.0b013e318263da2f

5. Kayaleh R, Krzyston H, Rishi A et al (2020) Transarterial radioembolization treatment of pancreatic cancer patients with liver-dominant metastatic disease using yttrium-90 glass microspheres: a single-institution retrospective study. J Vasc Interv Radiol 31:1060-1068. https://doi.org/10. 1016/j.jvir.2019.11.037

6. Elbanna KY, Jang HJ, Kim TK (2020) Imaging diagnosis and staging of pancreatic ductal adenocarcinoma: a comprehensive review. Insights Imaging 11(1):58. https://doi.org/10.1186/s13244-020-00861-y

7. Sakuhara Y, Kodama Y, Abo D et al (2008) Evaluation of the vascular supply to regions of the pancreas on CT during arteriography. Abdom Imaging 33:563-570. https://doi.org/10.1007/s00261-007-9342-x

8. Ishigaki S, Itoh S, Satake H, Ota T, Ishigaki T (2007) CT depiction of small arteries in the pancreatic head: assessment using coronal reformatted images with 16-channel multislice CT. Abdom Imaging 32:215-223. https://doi.org/10.1007/s00261-006-9042-y

9. Shi YJ, Zhang XP, Sun YS et al (2016) Spectral CT in the demonstration of the pancreatic arteries and their branches: a comparison with conventional CT. Medicine (Baltimore) 95:e2823. https://doi.org/10.1097/MD. 0000000000002823

10. Okahara M, Mori H, Kiyosue H, Yamada Y, Sagara Y, Matsumoto S (2010) Arterial supply to the pancreas; variations and cross-sectional anatomy. Abdom Imaging 35:134-142. https://doi.org/10.1007/s00261-009-9581-0

11. Macchi $\bigvee$, Picardi EEE, Porzionato A et al (2017) Anatomo-radiological patterns of pancreatic vascularization, with surgical implications: clinical and anatomical study. Clin Anat 30:614-624. https://doi.org/10.1002/ca.22885

12. Kim TM, Choi YH, Cheon JE et al (2019) Optimal kiloelectron volt for noise-optimized virtual monoenergetic images of dual-energy pediatric abdominopelvic computed tomography: preliminary results. Korean J Radiol 20:283-294. https://doi.org/10.3348/kjr.2017.0507

13. Rassouli N, Etesami M, Dhanantwari A, Rajiah P (2017) Detectorbased spectral CT with a novel dual-layer technology: principles and applications. Insights Imaging 8(6):589-598. https://doi.org/10.1007/ s13244-017-0571-4

14. Beer L, Toepker M, Ba-Ssalamah A et al (2019) Objective and subjective comparison of virtual monoenergetic vs. polychromatic images in patients with pancreatic ductal adenocarcinoma. Eur Radiol 29:36173625. https://doi.org/10.1007/s00330-019-06116-9
15. Szuák A Németh K, Korom C et al (2018) Pancreaticoduodenal arterial arcades: their dominance and variations-their potential clinical relevance. Clin Anat 31:544-550. https://doi.org/10.1002/ca.23063

16. Awai $K$, Inoue M, Yagyu Y et al (2004) Moderate versus high concentration of contrast material for aortic and hepatic enhancement and tumor-toliver contrast at multi-detector row CT. Radiology 233:682-688. https:// doi.org/10.1148/radiol.2333031617

17. Adam SZ, Rabinowich A, Kessner R, Blachar A (2021) Spectral CT of the abdomen: where are we now? Insights Imaging 12(1):138. https://doi. org/10.1186/s13244-021-01082-7

18. Ellmann S, Kammerer F, Allmendinger T et al (2018) Advanced modeled iterative reconstruction (ADMIRE) facilitates radiation dose reduction in abdominal CT. Acad Radiol 25:1277-1284. https://doi.org/10.1016/j.acra. 2018.01.014

19. Woisetschläger M, Henriksson L, Bartholomae W, Gasslander T, Björnsson B, Sandström P (2020) Iterative reconstruction algorithm improves the image quality without affecting quantitative measurements of computed tomography perfusion in the upper abdomen. Eur J Radiol 7:100243. https://doi.org/10.1016/j.ejro.2020.100243

20. Kimura W, Nagai H (1995) Study of surgical anatomy for duodenum-preserving resection of the head of the pancreas. Ann Surg 221(4):359-363. https://doi.org/10.1097/00000658-199504000-00005

21. Tanaka T, Sakaguchi H, Anai H et al (2004) Catheter position for adequate intra-arterial chemotherapy for advanced pancreatic cancer: evaluation with CT during arterial injection of contrast material. J Vasc Interv Radiol 15:1089-1097. https://doi.org/10.1097/01.RVI.0000131220.07444.7B

22. Lin $Y$, Yang $X$, Chen $Z$ et al (2012) Demonstration of the dorsal pancreatic artery by CTA to facilitate superselective arterial infusion of stem cells into the pancreas. Eur J Radiol 81(3):461-465. https://doi.org/10.1016/j.ejrad. 2010.12.077

23. Li J, Wang YH, Zheng FL et al (2021) Feasibility of utilizing ultra-low-dose contrast medium for pancreatic artery depiction using the combination of advanced virtual monoenergetic imaging and high-concentration contrast medium: an intra-patient study. Insights Imaging 12(1):166. https://doi.org/10.1186/s13244-021-01079-2

24. Bertelli E, Di Gregorio F, Bertelli L, Orazioli D, Bastianini A (1997) The arterial blood supply of the pancreas: a review. IV. The anterior inferior and posterior pancreaticoduodenal aa., and minor sources of blood supply for the head of the pancreas. An anatomical review and radiologic study. Surg Radiol Anat 19:203-212

25. Doppman JL, Miller DL, Chang R, Shawker TH, Gorden P, Norton JA (1991) Insulinomas: localization with selective intraarterial injection of calcium. Radiology 178:237-241. https://doi.org/10.1148/radiology.178.1.1984311

\section{Publisher's Note}

Springer Nature remains neutral with regard to jurisdictional claims in published maps and institutional affiliations. 